\title{
Complete $q$ th moment convergence for arrays of random variables
}

Soo Hak Sung*

\section{"Correspondence:}

sungsh@pcu.ac.kr

Department of Applied

Mathematics, Pai Chai University,

Taejon, 302-735, South Korea

\begin{abstract}
Let $\left\{X_{n i}, 1 \leq i \leq n, n \geq 1\right\}$ be an array of random variables with $E X_{n i}=0$ and $E\left|X_{n i}\right|^{q}<\infty$ for some $q \geq 1$. For any sequences $\left\{a_{n}, n \geq 1\right\}$ and $\left\{b_{n}, n \geq 1\right\}$ of positive real numbers, sets of sufficient conditions are given for complete $q$ th moment convergence of the form $\sum_{n=1}^{\infty} b_{n} a_{n}^{-q} E\left(\max _{1 \leq k \leq n}\left|\sum_{i=1}^{k} x_{n i}\right|-\epsilon a_{n}\right)_{+}^{q}<\infty, \forall \epsilon>0$, where $x_{+}=\max \{x, 0\}$. From these results, we can easily obtain some known results on complete qth moment convergence.
\end{abstract}

Keywords: complete convergence; complete moment convergence; $L^{9}$-convergence; dependent random variables

\section{Introduction}

The concept of complete convergence was introduced by Hsu and Robbins [1]. A sequence $\left\{X_{n}, n \geq 1\right\}$ of random variables is said to converge completely to the constant $\theta$ if

$$
\sum_{n=1}^{\infty} P\left(\left|X_{n}-\theta\right|>\epsilon\right)<\infty \quad \text { for all } \epsilon>0 .
$$

Hsu and Robbins [1] proved that the sequence of arithmetic means of i.i.d. random variables converges completely to the expected value if the variance of the summands is finite. Erdös [2] proved the converse.

The result of Hsu, Robbins, and Erdös has been generalized and extended in several directions. Baum and Katz [3] proved that if $\left\{X_{n}, n \geq 1\right\}$ is a sequence of i.i.d. random variables with $E X_{1}=0, E\left|X_{1}\right|^{p t}<\infty(1 \leq p<2, t \geq 1)$ is equivalent to

$$
\sum_{n=1}^{\infty} n^{t-2} P\left(\left|\sum_{i=1}^{n} X_{i}\right|>\epsilon n^{1 / p}\right)<\infty \quad \text { for all } \epsilon>0 .
$$

Chow [4] generalized the result of Baum and Katz [3] by showing the following complete moment convergence. If $\left\{X_{n}, n \geq 1\right\}$ is a sequence of i.i.d. random variables with $E X_{1}=0$ and $E\left(\left|X_{1}\right|^{p t}+\left|X_{1}\right| \log \left(1+\left|X_{1}\right|\right)\right)<\infty$ for some $0<p<2, t \geq 1$, and $p t \geq 1$, then

$$
\sum_{n=1}^{\infty} n^{t-2-1 / p} E\left(\max _{1 \leq k \leq n}\left|\sum_{i=1}^{k} X_{i}\right|-\epsilon n^{1 / p}\right)_{+}<\infty \quad \text { for all } \epsilon>0,
$$

where $x_{+}=\max \{x, 0\}$. Note that (1.2) implies (1.1). Li and Spătaru [5] gave a refinement of the result of Baum and Katz [3] as follows. Let $\left\{X_{n}, n \geq 1\right\}$ be a sequence of i.i.d. random

\section{照 Springer}

(c) 2013 Sung; licensee Springer. This is an Open Access article distributed under the terms of the Creative Commons Attribution License (http://creativecommons.org/licenses/by/2.0), which permits unrestricted use, distribution, and reproduction in any medium, provided the original work is properly cited. 
variables with $E X_{1}=0$, and let $0<p<2, t \geq 1, q>0$, and $p t \geq 1$. Then

$$
\begin{cases}E\left|X_{1}\right|^{q}<\infty & \text { if } q>p t \\ E\left|X_{1}\right|^{p t} \log \left(1+\left|X_{1}\right|\right)<\infty & \text { if } q=p t \\ E\left|X_{1}\right|^{p t}<\infty & \text { if } q<p t\end{cases}
$$

if and only if

$$
\int_{\epsilon}^{\infty} \sum_{n=1}^{\infty} n^{t-2} P\left(\left|\sum_{i=1}^{n} X_{i}\right|>x^{1 / q} n^{1 / p}\right) d x<\infty \quad \text { for all } \epsilon>0
$$

Recently, Chen and Wang [6] proved that for any $q>0$, any sequences $\left\{a_{n}, n \geq 1\right\}$ and $\left\{b_{n}, n \geq 1\right\}$ of positive real numbers and any sequence $\left\{Z_{n}, n \geq 1\right\}$ of random variables,

$$
\int_{\epsilon}^{\infty} \sum_{n=1}^{\infty} b_{n} P\left(\left|Z_{n}\right|>x^{1 / q} a_{n}\right) d x<\infty \quad \text { for all } \epsilon>0
$$

and

$$
\sum_{n=1}^{\infty} b_{n} a_{n}^{-q} E\left(\left|Z_{n}\right|-\epsilon a_{n}\right)_{+}^{q}<\infty \quad \text { for all } \epsilon>0,
$$

are equivalent. Therefore, if $\left\{X_{n}, n \geq 1\right\}$ is a sequence of i.i.d. random variables with $E X_{1}=0$ and $0<p<2, t \geq 1, q>0$, and $p t \geq 1$, then the moment condition (1.3) is equivalent to

$$
\sum_{n=1}^{\infty} n^{t-2-q / p} E\left(\left|\sum_{i=1}^{n} X_{i}\right|-\epsilon n^{1 / p}\right)_{+}^{q}<\infty \quad \text { for all } \epsilon>0 .
$$

When $q=1$, the complete $q$ th moment convergence (1.4) is reduced to complete moment convergence.

The complete $q$ th moment convergence for dependent random variables was established by many authors. Chen and Wang [7] showed that (1.3) and (1.4) are equivalent for $\varphi$-mixing random variables. Zhou and Lin [8] established complete $q$ th moment convergence theorems for moving average processes of $\varphi$-mixing random variables. Wu et al. [9] obtained complete $q$ th moment convergence results for arrays of rowwise $\rho$-mixing random variables.

The purpose of this paper is to provide sets of sufficient conditions for complete $q$ th moment convergence of the form

$$
\sum_{n=1}^{\infty} b_{n} a_{n}^{-q} E\left(\max _{1 \leq k \leq n}\left|\sum_{i=1}^{k} X_{n i}\right|-\epsilon a_{n}\right)_{+}^{q}<\infty \quad \text { for all } \epsilon>0,
$$

where $q \geq 1,\left\{a_{n}, n \geq 1\right\}$ and $\left\{b_{n}, n \geq 1\right\}$ are sequences of positive real numbers, and $\left\{X_{n i}, 1 \leq i \leq n, n \geq 1\right\}$ is an array of random variables satisfying Marcinkiewicz-Zygmund and Rosenthal type inequalities. When $q=1$, similar results were established by Sung [10]. From our results, we can easily obtain the results of Chen and Wang [7] and Wu et al. [9]. 


\section{Main results}

In this section, we give sets of sufficient conditions for complete $q$ th moment convergence (1.5). The following theorem gives sufficient conditions under the assumption that the array $\left\{X_{n i}, 1 \leq i \leq n, n \geq 1\right\}$ satisfies a Marcinkiewicz-Zygmund type inequality.

Theorem 2.1 Let $1 \leq q<2$ and let $\left\{X_{n i}, 1 \leq i \leq n, n \geq 1\right\}$ be an array of random variables with $E X_{n i}=0$ and $E\left|X_{n i}\right|^{q}<\infty$ for $1 \leq i \leq n$ and $n \geq 1$. Let $\left\{a_{n}, n \geq 1\right\}$ and $\left\{b_{n}, n \geq 1\right\}$ be sequences of positive real numbers. Suppose that the following conditions hold:

(i) for some $s(1 \leq q<s \leq 2)$, there exists a positive function $\alpha_{s}(x)$ such that

$$
\begin{aligned}
& E \max _{1 \leq k \leq n}\left|\sum_{i=1}^{k}\left(X_{n i}^{\prime}(x)-E X_{n i}^{\prime}(x)\right)\right|^{s} \\
& \quad \leq \alpha_{s}(n) \sum_{i=1}^{n} E\left|X_{n i}^{\prime}(x)\right|^{s} \text { for all } n \geq 1 \text { and } x>0,
\end{aligned}
$$

where $X_{n i}^{\prime}(x)=X_{n i} I\left(\left|X_{n i}\right| \leq x^{1 / q}\right)+x^{1 / q} I\left(X_{n i}>x^{1 / q}\right)-x^{1 / q} I\left(X_{n i}<-x^{1 / q}\right)$,

(ii) $\sum_{n=1}^{\infty} b_{n} a_{n}^{-s} \alpha_{s}(n) \sum_{i=1}^{n} E\left|X_{n i}\right|^{s} I\left(\left|X_{n i}\right| \leq a_{n}\right)<\infty$,

(iii) $\sum_{n=1}^{\infty} b_{n} a_{n}^{-q}\left(1+\alpha_{s}(n)\right) \sum_{i=1}^{n} E\left|X_{n i}\right|^{q} I\left(\left|X_{n i}\right|>a_{n}\right)<\infty$,

(iv) $\sum_{i=1}^{n} E\left|X_{n i}\right| I\left(\left|X_{n i}\right|>a_{n}\right) / a_{n} \rightarrow 0$.

Then (1.5) holds.

Proof It is obvious that

$$
\begin{aligned}
& \sum_{n=1}^{\infty} b_{n} a_{n}^{-q} E\left(\max _{1 \leq k \leq n}\left|\sum_{i=1}^{k} X_{n i}\right|-\epsilon a_{n}\right)_{+}^{q} \\
& =\sum_{n=1}^{\infty} b_{n} a_{n}^{-q} \int_{0}^{\infty} P\left(\max _{1 \leq k \leq n}\left|\sum_{i=1}^{k} X_{n i}\right|>\epsilon a_{n}+x^{1 / q}\right) d x \\
& \quad \leq \sum_{n=1}^{\infty} b_{n} a_{n}^{-q}\left\{\int_{0}^{a_{n}^{q}} P\left(\max _{1 \leq k \leq n}\left|\sum_{i=1}^{k} X_{n i}\right|>\epsilon a_{n}\right) d x+\int_{a_{n}^{q}}^{\infty} P\left(\max _{1 \leq k \leq n}\left|\sum_{i=1}^{k} X_{n i}\right|>x^{1 / q}\right) d x\right\} \\
& :=I_{1}+I_{2} .
\end{aligned}
$$

We first show that $I_{1}<\infty$. For $1 \leq i \leq n$ and $n \geq 1$, define

$$
X_{n i}^{\prime}=X_{n i} I\left(\left|X_{n i}\right| \leq a_{n}\right)+a_{n} I\left(X_{n i}>a_{n}\right)-a_{n} I\left(X_{n i}<-a_{n}\right), \quad X_{n i}^{\prime \prime}=X_{n i}-X_{n i}^{\prime} .
$$

Then we have by $E X_{n i}=0$, Markov's inequality, and (i) that

$$
\begin{aligned}
& P\left(\max _{1 \leq k \leq n}\left|\sum_{i=1}^{k} X_{n i}\right|>\epsilon a_{n}\right) \\
& \quad=P\left(\max _{1 \leq k \leq n}\left|\sum_{i=1}^{k}\left(X_{n i}^{\prime}-E X_{n i}^{\prime}+X_{n i}^{\prime \prime}-E X_{n i}^{\prime \prime}\right)\right|>\epsilon a_{n}\right) \\
& \quad \leq P\left(\max _{1 \leq k \leq n}\left|\sum_{i=1}^{k}\left(X_{n i}^{\prime}-E X_{n i}^{\prime}\right)\right|>\epsilon a_{n} / 2\right)+P\left(\max _{1 \leq k \leq n}\left|\sum_{i=1}^{k}\left(X_{n i}^{\prime \prime}-E X_{n i}^{\prime \prime}\right)\right|>\epsilon a_{n} / 2\right)
\end{aligned}
$$




$$
\begin{aligned}
\leq & 2^{s} \epsilon^{-s} a_{n}^{-s} E \max _{1 \leq k \leq n}\left|\sum_{i=1}^{k}\left(X_{n i}^{\prime}-E X_{n i}^{\prime}\right)\right|^{s}+2 \epsilon^{-1} a_{n}^{-1} E \max _{1 \leq k \leq n}\left|\sum_{i=1}^{k}\left(X_{n i}^{\prime \prime}-E X_{n i}^{\prime \prime}\right)\right| \\
\leq & 2^{s} \epsilon^{-s} a_{n}^{-s} \alpha_{s}(n) \sum_{i=1}^{n} E\left|X_{n i}^{\prime}\right|^{s}+4 \epsilon^{-1} a_{n}^{-1} \sum_{i=1}^{n} E\left|X_{n i}^{\prime \prime}\right| \\
\leq & 2^{s} \epsilon^{-s} a_{n}^{-s} \alpha_{s}(n) \sum_{i=1}^{n}\left(E\left|X_{n i}\right|^{s} I\left(\left|X_{n i}\right| \leq a_{n}\right)+a_{n}^{s} P\left(\left|X_{n i}\right|>a_{n}\right)\right) \\
& +4 \epsilon^{-1} a_{n}^{-1} \sum_{i=1}^{n} E\left|X_{n i}\right| I\left(\left|X_{n i}\right|>a_{n}\right) \\
\leq & 2^{s} \epsilon^{-s} a_{n}^{-s} \alpha_{s}(n) \sum_{i=1}^{n} E\left|X_{n i}\right|^{s} I\left(\left|X_{n i}\right| \leq a_{n}\right)+2^{s} \epsilon^{-s} a_{n}^{-q} \alpha_{s}(n) \sum_{i=1}^{n} E\left|X_{n i}\right|^{q} I\left(\left|X_{n i}\right|>a_{n}\right) \\
& +4 \epsilon^{-1} a_{n}^{-q} \sum_{i=1}^{n} E\left|X_{n i}\right|^{q} I\left(\left|X_{n i}\right|>a_{n}\right) .
\end{aligned}
$$

It follows that

$$
\begin{aligned}
I_{1}= & \sum_{n=1}^{\infty} b_{n} P\left(\max _{1 \leq k \leq n}\left|\sum_{i=1}^{k} X_{n i}\right|>\epsilon a_{n}\right) \\
\leq & 2^{s} \epsilon^{-s} \sum_{n=1}^{\infty} b_{n} a_{n}^{-s} \alpha_{s}(n) \sum_{i=1}^{n} E\left|X_{n i}\right|^{s} I\left(\left|X_{n i}\right| \leq a_{n}\right) \\
& +\sum_{n=1}^{\infty} b_{n} a_{n}^{-q}\left(2^{s} \epsilon^{-s} \alpha_{s}(n)+4 \epsilon^{-1}\right) \sum_{i=1}^{n} E\left|X_{n i}\right|^{q} I\left(\left|X_{n i}\right|>a_{n}\right) .
\end{aligned}
$$

Hence $I_{1}<\infty$ by (ii) and (iii).

We next show that $I_{2}<\infty$. By the definition of $X_{n i}^{\prime}(x)$, we have that

$$
\begin{aligned}
& P\left(\max _{1 \leq k \leq n}\left|\sum_{i=1}^{k} X_{n i}\right|>x^{1 / q}\right) \\
& \quad \leq \sum_{i=1}^{n} P\left(\left|X_{n i}\right|>x^{1 / q}\right)+P\left(\max _{1 \leq k \leq n}\left|\sum_{i=1}^{k} X_{n i}^{\prime}(x)\right|>x^{1 / q}\right) .
\end{aligned}
$$

We also have by $E X_{n i}=0$ and (iv) that

$$
\begin{aligned}
& \sup _{x \geq a_{n}^{q}} \max _{1 \leq k \leq n} x^{-1 / q}\left|\sum_{i=1}^{k} E X_{n i}^{\prime}(x)\right| \\
& =\sup _{x \geq a_{n}^{q}} \max _{1 \leq k \leq n} x^{-1 / q}\left|\sum_{i=1}^{k} E\left(X_{n i}-X_{n i}^{\prime}(x)\right)\right| \\
& \leq \sup _{x \geq a_{n}^{q}} x^{-1 / q} \sum_{i=1}^{n} E\left|X_{n i}\right| I\left(\left|X_{n i}\right|>x^{1 / q}\right) \\
& \leq a_{n}^{-1} \sum_{i=1}^{n} E\left|X_{n i}\right| I\left(\left|X_{n i}\right|>a_{n}\right) \rightarrow 0 .
\end{aligned}
$$


Hence to prove that $I_{2}<\infty$, it suffices to show that

$$
\begin{aligned}
& I_{3}:=\sum_{n=1}^{\infty} b_{n} a_{n}^{-q} \sum_{i=1}^{n} \int_{a_{n}^{q}}^{\infty} P\left(\left|X_{n i}\right|>x^{1 / q}\right) d x<\infty, \\
& I_{4}:=\sum_{n=1}^{\infty} b_{n} a_{n}^{-q} \int_{a_{n}^{q}}^{\infty} P\left(\max _{1 \leq k \leq n}\left|\sum_{i=1}^{k}\left(X_{n i}^{\prime}(x)-E X_{n i}^{\prime}(x)\right)\right|>x^{1 / q} / 2\right) d x<\infty .
\end{aligned}
$$

If $x>a_{n}^{q}$, then $P\left(\left|X_{n i}\right|>x^{1 / q}\right)=P\left(\left|X_{n i}\right| I\left(\left|X_{n i}\right|>a_{n}\right)>x^{1 / q}\right)$ and so

$$
\begin{aligned}
\int_{a_{n}^{q}}^{\infty} P\left(\left|X_{n i}\right|>x^{1 / q}\right) d x & =\int_{a_{n}^{q}}^{\infty} P\left(\left|X_{n i}\right| I\left(\left|X_{n i}\right|>a_{n}\right)>x^{1 / q}\right) d x \\
& \leq \int_{0}^{\infty} P\left(\left|X_{n i}\right| I\left(\left|X_{n i}\right|>a_{n}\right)>x^{1 / q}\right) d x=E\left|X_{n i}\right|^{q} I\left(\left|X_{n i}\right|>a_{n}\right),
\end{aligned}
$$

which implies that

$$
I_{3} \leq \sum_{n=1}^{\infty} b_{n} a_{n}^{-q} \sum_{i=1}^{n} E\left|X_{n i}\right|^{q} I\left(\left|X_{n i}\right|>a_{n}\right) .
$$

Hence $I_{3}<\infty$ by (iii).

Finally, we show that $I_{4}<\infty$. We get by Markov's inequality and (i) that

$$
\begin{aligned}
I_{4} \leq & 2^{s} \sum_{n=1}^{\infty} b_{n} a_{n}^{-q} \int_{a_{n}^{q}}^{\infty} x^{-s / q} E \max _{1 \leq k \leq n}\left|\sum_{i=1}^{k}\left(X_{n i}^{\prime}(x)-E X_{n i}^{\prime}(x)\right)\right|^{s} d x \\
\leq & 2^{s} \sum_{n=1}^{\infty} b_{n} a_{n}^{-q} \alpha_{s}(n) \sum_{i=1}^{n} \int_{a_{n}^{q}}^{\infty} x^{-s / q} E\left|X_{n i}^{\prime}(x)\right|^{s} d x \\
= & 2^{s} \sum_{n=1}^{\infty} b_{n} a_{n}^{-q} \alpha_{s}(n) \sum_{i=1}^{n} \int_{a_{n}^{q}}^{\infty} x^{-s / q}\left(E\left|X_{n i}\right|^{s} I\left(\left|X_{n i}\right| \leq x^{1 / q}\right)+x^{s / q} P\left(\left|X_{n i}\right|>x^{1 / q}\right)\right) d x \\
= & 2^{s} \sum_{n=1}^{\infty} b_{n} a_{n}^{-q} \alpha_{s}(n) \sum_{i=1}^{n} E\left|X_{n i}\right|^{s} I\left(\left|X_{n i}\right| \leq a_{n}\right) \int_{a_{n}^{q}}^{\infty} x^{-s / q} d x \\
& +2^{s} \sum_{n=1}^{\infty} b_{n} a_{n}^{-q} \alpha_{s}(n) \sum_{i=1}^{n} \int_{a_{n}^{q}}^{\infty} x^{-s / q} E\left|X_{n i}\right|^{s} I\left(a_{n}<\left|X_{n i}\right| \leq x^{1 / q}\right) d x \\
& +2^{s} \sum_{n=1}^{\infty} b_{n} a_{n}^{-q} \alpha_{s}(n) \sum_{i=1}^{n} \int_{a_{n}^{q}}^{\infty} P\left(\left|X_{n i}\right|>x^{1 / q}\right) d x:=I_{5}+I_{6}+I_{7} .
\end{aligned}
$$

Using a simple integral and Fubini's theorem, we obtain that

$$
\begin{aligned}
I_{5} & =2^{s} \frac{q}{s-q} \sum_{n=1}^{\infty} b_{n} a_{n}^{-s} \alpha_{s}(n) \sum_{i=1}^{n} E\left|X_{n i}\right|^{s} I\left(\left|X_{n i}\right| \leq a_{n}\right), \\
I_{6} & =2^{s} \sum_{n=1}^{\infty} b_{n} a_{n}^{-q} \alpha_{s}(n) \sum_{i=1}^{n} \int_{a_{n}^{q}}^{\infty} x^{-s / q} E\left|X_{n i}\right|^{s} I\left(a_{n}<\left|X_{n i}\right| \leq x^{1 / q}\right) d x \\
& =2^{s} \frac{q}{s-q} \sum_{n=1}^{\infty} b_{n} a_{n}^{-q} \alpha_{s}(n) \sum_{i=1}^{n} E\left|X_{n i}\right|^{q} I\left(\left|X_{n i}\right|>a_{n}\right) .
\end{aligned}
$$


Similarly to $I_{3}$,

$$
I_{7} \leq 2^{s} \sum_{n=1}^{\infty} b_{n} a_{n}^{-q} \alpha_{s}(n) \sum_{i=1}^{n} E\left|X_{n i}\right|^{q} I\left(\left|X_{n i}\right|>a_{n}\right) .
$$

Hence $I_{4}<\infty$ by (ii) and (iii).

The next theorem gives sufficient conditions for complete $q$ th moment convergence (1.5) under the assumption that the array $\left\{X_{n i}, 1 \leq i \leq n, n \geq 1\right\}$ satisfies a Rosenthal type inequality.

Theorem 2.2 Let $q \geq 1$ and let $\left\{X_{n i}, 1 \leq i \leq n, n \geq 1\right\}$ be an array of random variables with $E X_{n i}=0$ and $E\left|X_{n i}\right|^{q}<\infty$ for $1 \leq i \leq n$ and $n \geq 1$. Let $\left\{a_{n}, n \geq 1\right\}$ and $\left\{b_{n}, n \geq 1\right\}$ be sequences of positive real numbers. Suppose that the following conditions hold:

(i) for some $s>\max \{2,2 q / r\}$ ( $r$ is the same as in $(v)$ ), there exist positive functions $\beta_{s}(x)$ and $\gamma_{s}(x)$ such that

$$
\begin{aligned}
& E \max _{1 \leq k \leq n}\left|\sum_{i=1}^{k}\left(X_{n i}^{\prime}(x)-E X_{n i}^{\prime}(x)\right)\right|^{s} \\
& \leq \beta_{s}(n) \sum_{i=1}^{n} E\left|X_{n i}^{\prime}(x)\right|^{s}+\gamma_{s}(n)\left(\sum_{i=1}^{n} E\left|X_{n i}^{\prime}(x)\right|^{2}\right)^{s / 2} \\
& \quad \text { for all } n \geq 1 \text { and } x>0,
\end{aligned}
$$

where $X_{n i}^{\prime}(x)=X_{n i} I\left(\left|X_{n i}\right| \leq x^{1 / q}\right)+x^{1 / q} I\left(X_{n i}>x^{1 / q}\right)-x^{1 / q} I\left(X_{n i}<-x^{1 / q}\right)$,

(ii) $\sum_{n=1}^{\infty} b_{n} a_{n}^{-s} \beta_{s}(n) \sum_{i=1}^{n} E\left|X_{n i}\right|^{s} I\left(\left|X_{n i}\right| \leq a_{n}\right)<\infty$,

(iii) $\sum_{n=1}^{\infty} b_{n} a_{n}^{-q}\left(1+\beta_{s}(n)\right) \sum_{i=1}^{n} E\left|X_{n i}\right|^{q} I\left(\left|X_{n i}\right|>a_{n}\right)<\infty$,

(iv) $\sum_{i=1}^{n} E\left|X_{n i}\right| I\left(\left|X_{n i}\right|>a_{n}\right) / a_{n} \rightarrow 0$,

(v) $\sum_{n=1}^{\infty} b_{n} \gamma_{s}(n)\left(\sum_{i=1}^{n} a_{n}^{-r} E\left|X_{n i}\right|^{r}\right)^{s / 2}<\infty$ for some $0<r \leq 2$.

Then (1.5) holds.

Proof The proof is similar to that of Theorem 2.1. As in the proof of Theorem 2.1,

$$
\begin{aligned}
& \sum_{n=1}^{\infty} b_{n} a_{n}^{-q} E\left(\max _{1 \leq k \leq n}\left|\sum_{i=1}^{k} X_{n i}\right|-\epsilon a_{n}\right)_{+}^{q} \\
& \quad \leq \sum_{n=1}^{\infty} b_{n} a_{n}^{-q}\left\{\int_{0}^{a_{n}^{q}} P\left(\max _{1 \leq k \leq n}\left|\sum_{i=1}^{k} X_{n i}\right|>\epsilon a_{n}\right) d x+\int_{a_{n}^{q}}^{\infty} P\left(\max _{1 \leq k \leq n}\left|\sum_{i=1}^{k} X_{n i}\right|>x^{1 / q}\right) d x\right\} \\
& \quad:=J_{1}+J_{2} .
\end{aligned}
$$

Similarly to $I_{1}$ in the proof of Theorem 2.1, we have by the $c_{r}$-inequality that

$$
\begin{aligned}
& P\left(\max _{1 \leq k \leq n}\left|\sum_{i=1}^{k} X_{n i}\right|>\epsilon a_{n}\right) \\
& \leq 2^{s} \epsilon^{-s} a_{n}^{-s} \beta_{s}(n) \sum_{i=1}^{n}\left(E\left|X_{n i}\right|^{s} I\left(\left|X_{n i}\right| \leq a_{n}\right)+a_{n}^{s} P\left(\left|X_{n i}\right|>a_{n}\right)\right)
\end{aligned}
$$




$$
\begin{aligned}
& +2^{s} \epsilon^{-s} a_{n}^{-s} \gamma_{s}(n)\left(\sum_{i=1}^{n} E\left|X_{n i}\right|^{2} I\left(\left|X_{n i}\right| \leq a_{n}\right)+a_{n}^{2} P\left(\left|X_{n i}\right|>a_{n}\right)\right)^{s / 2} \\
& +4 \epsilon^{-1} a_{n}^{-1} \sum_{i=1}^{n} E\left|X_{n i}\right| I\left(\left|X_{n i}\right|>a_{n}\right) \\
& \leq 2^{s} \epsilon^{-s} a_{n}^{-s} \beta_{s}(n) \sum_{i=1}^{n} E\left|X_{n i}\right|^{s} I\left(\left|X_{n i}\right| \leq a_{n}\right) \\
& +\left(2^{s} \epsilon^{-s} \beta_{s}(n)+4 \epsilon^{-1}\right) a_{n}^{-q} \sum_{i=1}^{n} E\left|X_{n i}\right|^{q} I\left(\left|X_{n i}\right|>a_{n}\right) \\
& +2^{3 s / 2-1} \epsilon^{-s} \gamma_{s}(n)\left(\sum_{i=1}^{n} a_{n}^{-r} E\left|X_{n i}\right|^{r} I\left(\left|X_{n i}\right| \leq a_{n}\right)\right)^{s / 2} \\
& +2^{3 s / 2-1} \epsilon^{-s} \gamma_{s}(n)\left(\sum_{i=1}^{n} a_{n}^{-r} E\left|X_{n i}\right|^{r} I\left(\left|X_{n i}\right|>a_{n}\right)\right)^{s / 2}
\end{aligned}
$$

Hence $J_{1}<\infty$ by (ii), (iii), and (v).

As in the proof of Theorem 2.1, to prove that $J_{2}<\infty$, it suffices to show that

$$
\begin{aligned}
& J_{3}:=\sum_{n=1}^{\infty} b_{n} a_{n}^{-q} \sum_{i=1}^{n} \int_{a_{n}^{q}}^{\infty} P\left(\left|X_{n i}\right|>x^{1 / q}\right) d x<\infty, \\
& J_{4}:=\sum_{n=1}^{\infty} b_{n} a_{n}^{-q} \int_{a_{n}^{q}}^{\infty} P\left(\max _{1 \leq k \leq n}\left|\sum_{i=1}^{k}\left(X_{n i}^{\prime}(x)-E X_{n i}^{\prime}(x)\right)\right|>x^{1 / q} / 2\right) d x<\infty .
\end{aligned}
$$

The proof of $J_{3}<\infty$ is same as that of $I_{3}$ in the proof of Theorem 2.1.

For $J_{4}$, we have by Markov's inequality and (i) that

$$
\begin{aligned}
J_{4} & \leq 2^{s} \sum_{n=1}^{\infty} b_{n} a_{n}^{-q} \int_{a_{n}^{q}}^{\infty} x^{-s / q} E \max _{1 \leq k \leq n}\left|\sum_{i=1}^{k}\left(X_{n i}^{\prime}(x)-E X_{n i}^{\prime}(x)\right)\right|^{s} d x \\
& \leq 2^{s} \sum_{n=1}^{\infty} b_{n} a_{n}^{-q} \int_{a_{n}^{q}}^{\infty} x^{-s / q}\left\{\beta_{s}(n) \sum_{i=1}^{n} E\left|X_{n i}^{\prime}(x)\right|^{s}+\gamma_{s}(n)\left(\sum_{i=1}^{n} E\left|X_{n i}^{\prime}(x)\right|^{2}\right)^{s / 2}\right\} d x \\
& :=J_{5}+J_{6} .
\end{aligned}
$$

Similarly to $I_{4}$ in the proof of Theorem 2.1 , we get that

$$
\begin{aligned}
J_{5} \leq & 2^{s} \frac{q}{s-q} \sum_{n=1}^{\infty} b_{n} a_{n}^{-s} \beta_{s}(n) \sum_{i=1}^{n} E\left|X_{n i}\right|^{s} I\left(\left|X_{n i}\right| \leq a_{n}\right) \\
& +2^{s}\left(\frac{q}{s-q}+1\right) \sum_{n=1}^{\infty} b_{n} a_{n}^{-q} \beta_{s}(n) \sum_{i=1}^{n} E\left|X_{n i}\right|^{q} I\left(\left|X_{n i}\right|>a_{n}\right) .
\end{aligned}
$$

Hence $J_{5}<\infty$ by (ii) and (iii). 
Finally, we show that $J_{6}<\infty$. By the $c_{r}$-inequality,

$$
\begin{aligned}
J_{6}= & 2^{s} \sum_{n=1}^{\infty} b_{n} a_{n}^{-q} \gamma_{s}(n) \int_{a_{n}^{q}}^{\infty} x^{-s / q}\left(\sum_{i=1}^{n} E\left|X_{n i}\right|^{2} I\left(\left|X_{n i}\right| \leq x^{1 / q}\right)+x^{2 / q} P\left(\left|X_{n i}\right|>x^{1 / q}\right)\right)^{s / 2} d x \\
\leq & 2^{3 s / 2-1} \sum_{n=1}^{\infty} b_{n} a_{n}^{-q} \gamma_{s}(n) \int_{a_{n}^{q}}^{\infty} x^{-s / q}\left(\sum_{i=1}^{n} E\left|X_{n i}\right|^{2} I\left(\left|X_{n i}\right| \leq x^{1 / q}\right)\right)^{s / 2} d x \\
& +2^{3 s / 2-1} \sum_{n=1}^{\infty} b_{n} a_{n}^{-q} \gamma_{s}(n) \int_{a_{n}^{q}}^{\infty}\left(\sum_{i=1}^{n} P\left(\left|X_{n i}\right|>x^{1 / q}\right)\right)^{s / 2} d x \\
\leq & 2^{3 s / 2-1} \sum_{n=1}^{\infty} b_{n} a_{n}^{-q} \gamma_{s}(n) \int_{a_{n}^{q}}^{\infty} x^{-s / q}\left(\sum_{i=1}^{n} E\left|X_{n i}\right|^{r} x^{(2-r) / q}\right)^{s / 2} d x \\
& +2^{3 s / 2-1} \sum_{n=1}^{\infty} b_{n} a_{n}^{-q} \gamma_{s}(n) \int_{a_{n}^{q}}^{\infty}\left(\sum_{i=1}^{n} x^{-r / q} E\left|X_{n i}\right|^{r / 2} d x\right. \\
= & 2^{3 s / 2} \frac{2 q}{r s-2 q} \sum_{n=1}^{\infty} b_{n} \gamma_{s}(n)\left(\sum_{i=1}^{n} a_{n}^{-r} E\left|X_{n i}\right|^{r}\right)^{s / 2} .
\end{aligned}
$$

Hence $J_{6}<\infty$ by (v).

Remark 2.1 Marcinkiewicz-Zygmund and Rosenthal type inequalities hold for dependent random variables as well as independent random variables.

(1) Let $\left\{X_{n i}, 1 \leq i \leq n, n \geq 1\right\}$ be an array of rowwise negatively associated random variables. Then, for $1<s \leq 2$, (2.1) holds for $\alpha_{s}(n)=2^{s} 2^{3-s}=8$. For $s>2$, (2.2) holds for $\beta_{s}(n)=2^{s} 2(15 s / \log s)^{s}$ and $\gamma_{s}(n)=2(15 s / \log s)^{s}$ (see Shao [11]). Note that $\alpha_{s}(n)$ and $\beta_{s}(n)$ are multiplied by the factor $2^{s}$ since $E\left|X_{n i}^{\prime}(x)-E X_{n i}^{\prime}(x)\right|^{s} \leq 2^{s} E\left|X_{n i}^{\prime}(x)\right|^{s}$.

(2) Let $\left\{X_{n i}, 1 \leq i \leq n, n \geq 1\right\}$ be an array of rowwise negatively orthant dependent random variables. By Corollary 2.2 of Asadian et al. [12] and Theorem 3 of Móricz [13], (2.1) holds for $\alpha_{s}(n)=C_{1}(\log n)^{s}$, and (2.2) holds for $\beta_{s}(n)=C_{2}(\log n)^{s}$ and $\gamma_{s}(n)=C_{2}(\log n)^{s}$, where $C_{1}$ and $C_{2}$ are constants depending only on $s$.

(3) Let $\left\{X_{n}, n \geq 1\right\}$ be a sequence of identically distributed $\varphi$-mixing random variables. Set $X_{n i}=X_{i}$ for $1 \leq i \leq n$ and $n \geq 1$. By Shao's [14] result, (2.2) holds for a constant function $\beta_{s}(x)$ and a slowly varying function $\gamma_{s}(x)$. In particular, if $\sum_{n=1}^{\infty} \varphi^{1 / 2}\left(2^{n}\right)<\infty$, then (2.2) holds for some constant functions $\beta_{s}(x)$ and $\gamma_{s}(x)$.

(4) Let $\left\{X_{n}, n \geq 1\right\}$ be a sequence of identically distributed $\rho$-mixing random variables. Set $X_{n i}=X_{i}$ for $1 \leq i \leq n$ and $n \geq 1$. By Shao's [15] result, (2.2) holds for some slowly varying functions $\beta_{s}(x)$ and $\gamma_{s}(x)$. In particular, if $\sum_{n=1}^{\infty} \rho^{2 / s}\left(2^{n}\right)<\infty$, then (2.2) holds for some constant functions $\beta_{s}(x)$ and $\gamma_{s}(x)$.

(5) Let $\left\{X_{n}, n \geq 1\right\}$ be a sequence of $\rho^{\prime \prime}$-mixing random variables. Set $X_{n i}=X_{i}$ for $1 \leq i \leq n$ and $n \geq 1$. By the result of Utev and Peligrad [16], (2.2) holds for some constant functions $\beta_{s}(x)$ and $\gamma_{s}(x)$.

\section{Corollaries}

In this section, we establish some complete $q$ th moment convergence results by using the results obtained in the previous section. 
Corollary 3.1 (Chen and Wang [7]) Let $\left\{X_{n}, n \geq 1\right\}$ be a sequence of identically distributed $\varphi$-mixing random variables with $E X_{1}=0$, and let $t \geq 1,0<p<2, q \geq 1$, and $p t \geq 1$. Assume that (1.3) holds. Furthermore, suppose that

$$
\sum_{n=1}^{\infty} \varphi^{1 / 2}\left(2^{n}\right)<\infty
$$

if $t=1$ and $\max \{q, p t\}<2$. Then

$$
\sum_{n=1}^{\infty} n^{t-2-q / p} E\left(\max _{1 \leq k \leq n}\left|\sum_{i=1}^{k} X_{i}\right|-\epsilon n^{1 / p}\right)_{+}^{q}<\infty \quad \text { for all } \epsilon>0 .
$$

Proof Let $a_{n}=n^{1 / p}$ and $b_{n}=n^{t-2}$ for $n \geq 1$, and let $X_{n i}=X_{i}$ for $1 \leq i \leq n$ and $n \geq 1$. Then, for $s \geq 2,(2.2)$ holds for a constant function $\beta_{s}(x)$ and a slowly varying function $\gamma_{s}(x)$ (see Remark 2.1(3)). Under the additional condition that $\sum_{n=1}^{\infty} \varphi^{1 / 2}\left(2^{n}\right)<\infty$, (2.2) holds for some constant functions $\beta_{s}(x)$ and $\gamma_{s}(x)$. In particular, for $s=2$, (2.1) holds for a constant function $\alpha_{s}(x)$ under this additional condition.

By a standard method, we have that

$$
\begin{aligned}
& \sum_{n=1}^{\infty} n^{t-1-s / p} E\left|X_{1}\right|^{s} I\left(\left|X_{1}\right| \leq n^{1 / p}\right) \leq C E\left|X_{1}\right|^{p t} \quad \text { if } p t<s, \\
& \sum_{n=1}^{\infty} n^{t-1-q \mid p} E\left|X_{1}\right|^{q} I\left(\left|X_{1}\right|>n^{1 / p}\right) \leq \begin{cases}C E\left|X_{1}\right|^{q} & \text { if } q>p t, \\
C E\left|X_{1}\right|^{p t} \log \left(1+\left|X_{1}\right|\right) & \text { if } q=p t, \\
C E\left|X_{1}\right|^{p t} & \text { if } q<p t,\end{cases} \\
& n^{1-1 / p} E\left|X_{1}\right| I\left(\left|X_{1}\right|>n^{1 / p}\right) \leq n^{1-t} E\left|X_{1}\right|^{p t} I\left(\left|X_{1}\right|>n^{1 / p}\right) \quad \text { if } p t \geq 1,
\end{aligned}
$$

where $C$ is a positive constant which is not necessarily the same one in each appearance. Hence, the conditions (i)-(iv) of Theorem 2.2 hold if we take $s>\max \{p t, 2,2 q / r\}$. Under the additional conditions that $\max \{q, p t\}<2$ and $\sum_{n=1}^{\infty} \varphi^{1 / 2}\left(2^{n}\right)<\infty$, all conditions of Theorem 2.1 hold if we take $s=2$. Therefore, the result follows from Theorems 2.1 and 2.2 if we only show that the condition (v) of Theorem 2.2 holds when $t>1$ or $\max \{q, p t\} \geq 2$. To do this, we take $r=2$ if $\max \{q, p t\} \geq 2$ and $r=\max \{q, p t\}$ if $\max \{q, p t\}<2$. If $t>1$ or $\max \{q, p t\} \geq 2$, then $r>p$ and so we can choose $s>2$ large enough such that $t-1+(1-r / p) s / 2<0$. Then

$$
\sum_{n=1}^{\infty} b_{n} \gamma_{s}(n)\left(\sum_{i=1}^{n} a_{n}^{-r} E\left|X_{n i}\right|^{r}\right)^{s / 2}=\left(E\left|X_{1}\right|^{r}\right)^{s / 2} \sum_{n=1}^{\infty} \gamma_{s}(n) n^{t-2+(1-r / p) s / 2}<\infty
$$

Hence the condition (v) of Theorem 2.2 holds.

Let $\left\{\Psi_{n}(x), n \geq 1\right\}$ be a sequence of positive even functions satisfying

$$
\frac{\Psi_{n}(|x|)}{|x|^{q}} \uparrow \quad \text { and } \quad \frac{\Psi_{n}(|x|)}{|x|^{s}} \downarrow \quad \text { as }|x| \uparrow
$$

for some $1 \leq q<s$. 
Corollary 3.2 Let $\left\{\Psi_{n}(x), n \geq 1\right\}$ be a sequence of positive even functions satisfying (3.1) for some $1 \leq q<s \leq 2$. Let $\left\{X_{n i}, 1 \leq i \leq n, n \geq 1\right\}$ be an array of random variables satisfying $E X_{n i}=0$ for $1 \leq i \leq n$ and $n \geq 1$, and (2.1) for some constant function $\alpha_{s}(x)$. Let $\left\{a_{n}, n \geq 1\right\}$ and $\left\{b_{n}, n \geq 1\right\}$ be sequences of positive real numbers. Suppose that the following conditions hold:

(i) $\sum_{n=1}^{\infty} b_{n} \sum_{i=1}^{n} E \Psi_{i}\left(\left|X_{n i}\right|\right) / \Psi_{i}\left(a_{n}\right)<\infty$,

(ii) $\sum_{i=1}^{n} E \Psi_{i}\left(\left|X_{n i}\right|\right) / \Psi_{i}\left(a_{n}\right) \rightarrow 0$.

Then (1.5) holds.

Proof First note by $\Psi_{i}(|x|) /|x|^{q} \uparrow$ that $\Psi_{i}(|x|)$ is an increasing function. Since $\Psi_{i}(|x|) /|x|^{s} \downarrow$,

$$
\frac{\left|X_{n i}\right|^{s} I\left(\left|X_{n i}\right| \leq a_{n}\right)}{a_{n}^{s}} \leq \frac{\Psi_{i}\left(\left|X_{n i}\right| I\left(\left|X_{n i}\right| \leq a_{n}\right)\right)}{\Psi_{i}\left(a_{n}\right)} \leq \frac{\Psi_{i}\left(\left|X_{n i}\right|\right)}{\Psi_{i}\left(a_{n}\right)} .
$$

Since $q \geq 1$ and $\Psi_{i}(|x|) /|x|^{q} \uparrow$,

$$
\frac{\left|X_{n i}\right| I\left(\left|X_{n i}\right|>a_{n}\right)}{a_{n}} \leq \frac{\left|X_{n i}\right|^{q} I\left(\left|X_{n i}\right|>a_{n}\right)}{a_{n}^{q}} \leq \frac{\Psi_{i}\left(\left|X_{n i}\right| I\left(\left|X_{n i}\right|>a_{n}\right)\right)}{\Psi_{i}\left(a_{n}\right)} \leq \frac{\Psi_{i}\left(\left|X_{n i}\right|\right)}{\Psi_{i}\left(a_{n}\right)} .
$$

It follows that all conditions of Theorem 2.1 are satisfied and so the result follows from Theorem 2.1.

Corollary 3.3 Let $\left\{\Psi_{n}(x), n \geq 1\right\}$ be a sequence of positive even functions satisfying (3.1) for some $q \geq 1$ and $s>\max \{2, q\}$. Let $\left\{X_{n i}, 1 \leq i \leq n, n \geq 1\right\}$ be an array of random variables satisfying $E X_{n i}=0$ for $1 \leq i \leq n$ and $n \geq 1$, and (2.2) for some constant functions $\beta_{s}(x)$ and $\gamma_{s}(x)$. Let $\left\{a_{n}, n \geq 1\right\}$ and $\left\{b_{n}, n \geq 1\right\}$ be sequences of positive real numbers. Suppose that the following conditions hold:

(i) $\sum_{n=1}^{\infty} b_{n} \sum_{i=1}^{n} E \Psi_{i}\left(\left|X_{n i}\right|\right) / \Psi_{i}\left(a_{n}\right)<\infty$,

(ii) $\sum_{i=1}^{n} E \Psi_{i}\left(\left|X_{n i}\right|\right) / \Psi_{i}\left(a_{n}\right) \rightarrow 0$,

(iii) $\sum_{n=1}^{\infty} b_{n}\left(\sum_{i=1}^{n} a_{n}^{-2} E\left|X_{n i}\right|^{2}\right)^{s / 2}<\infty$.

Then (1.5) holds.

Proof The proof is similar to that of Corollary 3.2. By the proof of Corollary 3.2 and the condition (iii), all conditions of Theorem 2.2 are satisfied and so the result follows from Theorem 2.2.

Remark 3.1 When $b_{n}=1$ for $n \geq 1$, the condition (i) of Corollaries 3.2 and 3.3 is reduced to the condition $\sum_{n=1}^{\infty} \sum_{i=1}^{n} E \Psi_{i}\left(\left|X_{n i}\right|\right) / \Psi_{i}\left(a_{n}\right)<\infty$, and so the condition (ii) of Corollaries 3.2 and 3.3 follows from this reduced condition. For a sequence of $\rho^{*}$-mixing random variables, (2.1) holds for some constant function $\alpha_{s}(x)$ if $s=2$, and (2.2) holds for some constant functions $\beta_{s}(x)$ and $\gamma_{s}(x)$ if $s>2$ (see Remark 2.1(5)). Wu et al. [9] proved Corollaries 3.2 and 3.3 when $b_{n}=1$ for $n \geq 1$, and $\left\{X_{n i}\right\}$ is an array of rowwise $\rho^{*}$-mixing random variables. 
Received: 9 October 2012 Accepted: 3 January 2013 Published: 17 January 2013

\section{References}

1. Hsu, PL, Robbins, H: Complete convergence and the law of large numbers. Proc. Natl. Acad. Sci. USA 33, 25-31 (1947)

2. Erdös, P: On a theorem of Hsu and Robbins. Ann. Math. Stat. 20, 286-291 (1949)

3. Baum, LE, Katz, M: Convergence rates in the law of large numbers. Trans. Am. Math. Soc. 120, 108-123 (1965)

4. Chow, YS: On the rate of moment convergence of sample sums and extremes. Bull. Inst. Math. Acad. Sin. 16, 177-201 (1988)

5. Li, D, Spătaru, A: Refinement of convergence rates for tail probabilities. J. Theor. Probab. 18, 933-947 (2005)

6. Chen, PY, Wang, DC: Convergence rates for probabilities of moderate deviations for moving average processes. Acta Math. Sin. 24, 611-622 (2008)

7. Chen, PY, Wang, DC: Complete moment convergence for sequences of identically distributed $\varphi$-mixing random variables. Acta Math. Sin. 26, 679-690 (2010)

8. Zhou, XC, Lin, JG: Complete $q$-moment convergence of moving average processes under $\varphi$-mixing assumption. J. Math. Res. Expo. 31, 687-697 (2011)

9. $\mathrm{Wu}, \mathrm{Y}$, Wang, C, Volodin, A: Limiting behavior for arrays of rowwise $\rho^{*}$-mixing random variables. Lith. Math. J. 52, 214-221 (2012)

10. Sung, SH: Moment inequalities and complete moment convergence. J. Inequal. Appl. 2009, Article ID 271265 (2009)

11. Shao, QM: A comparison theorem on moment inequalities between negatively associated and independent random variables. J. Theor. Probab. 13, 343-356 (2000)

12. Asadian, N, Fakoor, V, Bozorgnia, A: Rosenthal's type inequalities for negatively orthant dependent random variables. J. Iran. Stat. Soc. 5, 69-75 (2006)

13. Móricz, F: Moment inequalities and the strong laws of large numbers. Z. Wahrscheinlichkeitstheor. Verw. Geb. 35 299-314 (1976)

14. Shao, QM: A moment inequality and its applications. Acta Math. Sin. Chin. Ser. 31, 736-747 (1988)

15. Shao, QM: Maximal inequalities for partial sums of $\rho$-mixing sequences. Ann. Probab. 23, 948-965 (1995)

16. Utev, S, Peligrad, M: Maximal inequalities and an invariance principle for a class of weakly dependent random variables. J. Theor. Probab. 16, 101-115 (2003)

doi:10.1186/1029-242X-2013-24

Cite this article as: Sung: Complete $q$ th moment convergence for arrays of random variables. Journal of Inequalities and Applications 2013 2013:24

\section{Submit your manuscript to a SpringerOpen ${ }^{\ominus}$ journal and benefit from:}

- Convenient online submission

Rigorous peer review

- Immediate publication on acceptance

- Open access: articles freely available online

- High visibility within the field

- Retaining the copyright to your article 